\title{
ON THE VIRASORO ALGEBRA AS REDUCED POISSON SUBMANIFOLD OF A KAC-MOODY ALGEBRA ON THE CIRCLE
}

\author{
GLORIA MARI BEFFA
}

(Communicated by Peter Li)

\begin{abstract}
In this paper we show how the Lie-Poisson structure on the dual of the Virasoro algebra can be obtained through a standard Poisson reduction process performed on a Kac-Moody algebra on the circle. We use the geometrical idea of the process to establish some relation between transverse structures on both Poisson manifolds.
\end{abstract}

\section{INTRODUCTION}

For a long time it has been known that the Korteweg-de Vries equation $u_{t}=$ $6 u u_{x}-u_{x x x}$ can be written as a Hamiltonian system with respect to two different Poisson structures (see Lax's paper [9], or Adler's [1]). This bi-Hamiltonian character gives rise to the definition of a sequence of Hamiltonian structures and commuting Hamiltonian operators which can be generated by recursion (see, for example, [2]). The second Hamiltonian structure in this hierarchy coincides with the canonical Lie-Poisson structure on the dual of the Virasoro algebra. Later, in an attempt to generalize this property of the $\mathrm{KdV}$ equation, Adler [1] defined a family of second Hamiltonian structures with respect to which the generalized $\mathrm{KdV}$ equations could also be written as Hamiltonian systems. Jacobi's identity for these brackets was proved by Gelfand and Dikii in [5]. These Poisson structures are called second Hamiltonian $K d V$ structures or Gelfand-Dikii brackets, and they are defined on the space of $n$ th-order scalar differential operators (or Lax operators). In the case of second-order operators with a vanishing first-order term the Gelfand-Dikii bracket coincides with the Lie-Poisson structure on the dual of the Virasoro algebra (it is also the only case in which the second Hamiltonian $\mathrm{KdV}$ structure is linear).

Drinfeld and Sokolov established a connection between Lie algebras and the Gelfand-Dikii brackets in their paper Lie algebras and equations of KdV type [4]. They showed how Gelfand-Dikii brackets could be obtained from the canonical Lie-Poisson structure on a Kac-Moody algebra on the circle associated to a semisimple Lie group $G$ following certain reduction process. The case $G=$ $\operatorname{SL}(2, \mathbf{R})$ coincides with the Virasoro algebra.

Received by the editors October 18, 1992 and, in revised form, February 23, 1993.

1991 Mathematics Subject Classification. Primary 53C80; Secondary 53C57. 
In this paper we offer a simpler explanation of their result for the Virasoro algebra case using a standard geometrical reduction process. A similar idea has also been used by Casati and Pedroni in [3]. In their paper, Casati and Pedroni show a rather algebraic way to obtain classical equations which are Hamiltonian systems with respect to Gelfand-Dikii brackets, using Drinfeld and Sokolov reduction. For that, they apply a modified theorem by Marsden and Ratiu about Poisson reduction on bi-Hamiltonian manifolds. In this paper we also use a theorem by Marsden and Ratiu which can be found in [10]. Although as stated in [10] the theorem is valid for finite-dimensional Poisson manifolds and does not include the case of the dual of the Virasoro algebra (a Fréchet manifold), a minor argument would allow us to apply the theorem directly. Instead of doing so, we prefer to repeat the geometric arguments in [10] so that the reader may be made more familiar with the geometry of these manifolds. In this way, we prove the existence of a reduced Poisson bracket on a certain quotient manifold. Rather than showing how to obtain Hamiltonian equations on this quotient, we show that, furthermore, the quotient manifold is Poisson-isomorphic to the dual of the Virasoro algebra (Theorem 2.1). This procedure is geometrically clearer than that in [4] since the algebra of pseudodifferential operators is not used. It is also a more appropriate approach to work in the geometrical properties of these brackets. For example, it allows the establishment of some relation between transverse structures for the Lie-Poisson brackets on the dual of the Kac-Moody algebra on the circle associated to $\operatorname{SL}(2, \mathbf{R})$ and on the dual of the Virasoro algebra. We comment on this relation at the end of $\S 2$.

\section{KaC-Moody algebras on the CirCle AND their PoisSON STRUCTUReS INHERITED FROM THE CORRESPONDING CURRENT ALGEBRA}

Let $G$ be a real semisimple Lie group and $g$ its Lie algebra. Let $\widetilde{G}=$ $C^{\infty}\left(S^{1} ; G\right)$ be the group of smooth functions on the circle with values on $G$ (or group of loops) with the pointwise product. $\widetilde{G}$ is also called the current group. Its Lie algebra $\tilde{g}=C^{\infty}\left(S^{1} ; g\right)$ is called the current algebra, and it has the pointwise Lie bracket as Lie algebra structure. Denote by $B$ the Killing form of the Lie algebra $g$, and define the following scalar product on $\tilde{g}$ :

$$
(L, M)=\int_{0}^{1} B(L(\theta), M(\theta)) d \theta .
$$

This scalar product enables us to embed $\tilde{g}$ in its dual space. Next, define

$$
\omega(L, M)=\int_{0}^{1} B\left(L(\theta), \frac{d M(\theta)}{d \theta}\right) d \theta .
$$

It is easy to check that

$$
\omega([L, M], N)+\omega([M, N], L)+\omega([N, L], M)=0
$$

for all $L, M, N \in \tilde{g}$. That is, $\omega$ is a cocycle for $\tilde{g}$ (see [13]). Define the Lie algebra $\hat{g}=\tilde{g} \oplus \mathbf{R}$ with Lie bracket

$$
[(L, s),(M, t)]=([L, M], \omega(L, M)) \text {. }
$$

$\hat{g}$ is called the central extension of $\tilde{g}$ obtained through $\omega$ (see [13]). In this particular case $\hat{g}$ is also called a Kac-Moody algebra on $S^{1}$.

Next we will state some of the known results about these structures. For their proofs see, for example, Pressley and Segal [13] or Reiman and Semenov [14]. 
Proposition 1.1. With the appropriate identifications the coadjoint representation of the algebra $\hat{g}$ is given by the formula

$$
\operatorname{ad}^{*}(M, t)(L, s)=\left([M, L]+s \frac{d M}{d \theta}, 0\right),
$$

for any $L, M \in \tilde{g}$ and any $t, s \in \mathbf{R}$. The center of $\hat{g}$ acts trivially on $\hat{g}^{*}$, and therefore the coadjoint action above can be derived from the action of $\widetilde{G}$ on $\hat{g}^{*}$. This action is given by the formula

$$
\operatorname{Ad}^{*}(g)(L, s)=\left(\operatorname{Ad}_{G}(g)(L)+s \frac{d g}{d \theta} g^{-1}, s\right),
$$

where $\operatorname{Ad}_{G}$ represents the adjoint action of $\widetilde{G}$ on $\tilde{g}$.

Because of (1.1) and (1.2), the space $\hat{g}^{*}$ is usually identified with the space of matrix differential operators of the form

$$
-s \frac{d}{d \theta}+L(\theta)
$$

where $L \in \tilde{g}^{*}$ and $s \in \mathbf{R}$. Under this identification the coadjoint action is simply the gauge action of $\widetilde{G}$ on the space of differential operators of the form (1.3). The coadjoint orbits correspond to the symplectic leaves of the usual Lie-Poisson structure of the algebra, which in this case is given by the formula

$$
\begin{aligned}
\{\mathscr{H}, \mathscr{L}\}(L, s) & =([d \mathscr{H}, d \mathscr{L}], L)+s \omega(d \mathscr{H}, d \mathscr{L}) \\
& =\int_{0}^{1} B\left(s \frac{d(d \mathscr{H})}{d \theta}+[d \mathscr{H}, d \mathscr{L}], L\right) d \theta \\
& =\int_{0}^{1} B\left(\operatorname{ad}^{*}(d \mathscr{H})(d \mathscr{L}, s), L\right) d \theta .
\end{aligned}
$$

From (1.2), we observe that the central parameter $s$ is fixed under the coadjoint action of the group. Therefore, the Kac-Moody algebra $\hat{g}$ stratifies into Poisson submanifolds corresponding to different values of the parameter; each one of these submanifolds is isomorphic to $\tilde{g}$ endowed with the Poisson bracket (1.4). We will focus our attention on the study of the hyperplane given by $s=1$. Let us denote it by $\hat{g}_{1}^{*}$. The Lie-Poisson structure on this hyperplane is referred to as a second Hamiltonian structure on $\hat{g}_{1}^{*}$.

From now on we will restrict ourselves to the case $G=\operatorname{SL}(2, \mathbf{R})$, although we will frequently point out what to do in the general case.

Given an operator $P=-\frac{d}{d \theta}+M(\theta)$ with $M \in C^{\infty}\left(S^{1} ; g\right)$, consider the associated differential problem $\frac{d \xi}{d \theta}=M \xi$. We will denote by $T(P)$ the monodromy matrix associated with $\frac{d \xi}{d \theta}=M \xi$ (that is, if $X$ is a fundamental matrix solution of $\frac{d \xi}{d \theta}=M \xi$, then by definition $\left.T(P)=X(0) X^{-1}(2 \pi)\right)$. The following known results give a brief description of the coadjoint orbits (Kirillov symplectic leaves) of the Poisson structure (1.4).

Theorem 1.1. The $\mathrm{SL}(2, \mathbf{R})$-conjugation class of the monodromy $T(P)$ completely determines the orbit of $P$.

Corollary 1.1. If $T(P)=I$, where $I$ is the identity matrix, then the zero matrix is in the coadjoint orbit of $P$ and the codimension of such an orbit is three. If 
$T(P)=-I$ then $-\frac{d}{d \theta}+\left(\begin{array}{cc}0 & -1 / 4 \\ 1 & 0\end{array}\right)$ is in the coadjoint orbit of $P=-\frac{d}{d \theta}+M(\theta)$ and the codimension of such an orbit is also three. Furthermore, the two orbits corresponding to the identity and to minus the identity are the only ones that have codimension three. Any other orbit has codimension one.

Proof. The first part is an immediate consequence of Theorem 1.1. To prove the second part we look at the expression of the coadjoint action (1.1) and observe that the codimension of the orbit of $P=-\frac{d}{d \theta}+M$ coincides with the number of independent periodic solutions of equation $\frac{d Y}{d \theta}+[Y, M]=0$. Any solution of this equation is of the form $Y=X(\theta) X_{0} X(\theta)^{-1}$, where $X_{0} \in g$ and $X$ is a fundamental matrix solution for $\frac{d \xi}{d \theta}=M \xi$. Therefore, $Y$ is periodic if and only if

$$
X_{0}=T(P) X_{0} T(P)^{-1} .
$$

Any matrix $T(P)$ either fixes the whole algebra (namely, when $T(P)=I$ or $T(P)=-I$ ) or only a 1-dimensional subalgebra (which would be generated by $T(P)-\operatorname{trace}(T(P)) I)$. Therefore, the codimension of a symplectic leaf will be three whenever the monodromy is $\pm I$ and one in the remaining cases.

In [14] it was shown that the conjugation class of $T(P)$ is the only invariant under the coadjoint action. Notice also that all the results described up to now can be formulated in exactly the same way if one replaces the group $\operatorname{SL}(2, \mathbf{R})$ by a general semisimple Lie group $G$.

To finish this section we will give an explicit formula in coordinates of the bracket (1.4) which will be used in $\S 2$.

Definitions 1.1. Let $X_{1}, X_{2}, X_{3}$ be generators of $\mathrm{sl}(2, \mathbf{R})$ given by

$$
X_{1}=\left(\begin{array}{cc}
\frac{1}{2} & 0 \\
0 & -\frac{1}{2}
\end{array}\right), \quad X_{2}=\left(\begin{array}{cc}
0 & \frac{1}{2} \\
-\frac{1}{2} & 0
\end{array}\right), \quad X_{3}=\left(\begin{array}{cc}
0 & \frac{1}{2} \\
\frac{1}{2} & 0
\end{array}\right) .
$$

On $\operatorname{sl}(2, \mathbf{R})$ consider the scalar product given by $(X, Y)=\operatorname{trace}(X, Y)$ and let $X_{1}^{*}, X_{2}^{*}, X_{3}^{*}$ be the dual elements for $X_{1}, X_{2}, X_{3}$ with respect to $($,$) .$ With this notation, any element $X \in C^{\infty}\left(S^{1} ; \mathrm{sl}(2, \mathbf{R})^{*}\right)$ can be written as $X=$ $x_{1} X_{1}^{*}+x_{2} X_{2}^{*}+x_{3} X_{3}^{*}$ for some $2 \pi$-periodic real-valued functions $x_{1}, x_{2}, x_{3}$.

Define $\varepsilon_{k}^{i}: \tilde{g} \rightarrow \mathbf{R}$ to be the $k$ th Fourier coefficient of the component $x_{i}$, for $i=1,2,3$; that is,

$$
\varepsilon_{k}^{i}(X)=\int_{0}^{2 \pi} \frac{1}{2 \pi} e^{-i k \theta} \operatorname{trace}\left(X X_{i}\right) d \theta
$$

$i=1,2,3$ and $k=\ldots,-2,-1,1,2, \ldots$. The family $\left\{\varepsilon_{i}^{k}\right\}_{k, i}$ provides a complete set of coordinates for $\tilde{g}_{1}^{*}$, and the gradient of $\varepsilon_{k}^{i}$ is given by $\frac{1}{2 \pi} e^{-i k \theta} X_{i} \in \tilde{g}$.

In these coordinates it is straightforward to check that the Lie-Poisson bracket 
(1.4) looks like

$$
\begin{aligned}
\left\{\varepsilon_{k}^{1}, \varepsilon_{d}^{2}\right\}(X) & =\int_{0}^{2 \pi} \frac{1}{[2 \pi]^{2}} e^{-i(k+d) \theta}\left[\operatorname{trace}\left(\left[X_{1}, X_{2}\right] X-i d X_{1} X_{2}\right)\right] d \theta \\
& =\frac{1}{2 \pi} \varepsilon_{k+d}^{3}(X) \\
\left\{\varepsilon_{k}^{1}, \varepsilon_{d}^{3}\right\}(X) & =\int_{0}^{2 \pi} \frac{1}{[2 \pi]^{2}} e^{-i(k+d) \theta}\left[\operatorname{trace}\left(\left[X_{1}, X_{3}\right] X-i d X_{1} X_{3}\right)\right] d \theta \\
& =\frac{1}{2 \pi} \varepsilon_{k+d}^{2}(X), \\
\left\{\varepsilon_{k}^{2}, \varepsilon_{d}^{3}\right\}(X) & =\int_{0}^{2 \pi} \frac{1}{[2 \pi]^{2}} e^{-i(k+d) \theta}\left[\operatorname{trace}\left(\left[X_{2}, X_{3}\right] X-i d X_{2} X_{3}\right)\right] d \theta \\
& =\frac{1}{2 \pi} \varepsilon_{k+d}^{1}(X)
\end{aligned}
$$

and

$$
\left\{\varepsilon_{k}^{1}, \varepsilon_{d}^{1}\right\}(X)=\frac{-i d}{4 \pi} \delta_{k+d}^{0}, \quad\left\{\varepsilon_{k}^{2}, \varepsilon_{d}^{2}\right\}(X)=\frac{i d}{4 \pi} \delta_{k+d}^{0}, \quad\left\{\varepsilon_{k}^{3}, \varepsilon_{d}^{3}\right\}(X)=\frac{-i d}{4 \pi} \delta_{k+d}^{0},
$$

where we have made use of the $\operatorname{sl}(2, \mathbf{R})$-commutation relations $\left[X_{1}, X_{2}\right]=X_{3}$, $\left[X_{1}, X_{3}\right]=X_{2},\left[X_{2}, X_{3}\right]=X_{1}$ and of the fact that $\operatorname{trace}\left(X_{i} X_{j}\right)=0$ whenever $i \neq j$ and $\operatorname{trace}\left(X_{1}^{2}\right)=-\operatorname{trace}\left(X_{2}^{2}\right)=\operatorname{trace}\left(X_{3}^{2}\right)=\frac{1}{2}$ and where $\delta_{i}^{j}$ is the Kronecker delta, that is, $\delta_{i}^{j}=0$ if $i \neq j$ and $\delta_{i}^{i}=1$ for all $i$.

\section{The Virasoro algebra as a Poisson submanifold of $\hat{g}_{1}^{*}$}

In this section we will describe a certain foliation on a submanifold $\mathscr{M}$ of $\hat{g}_{1}^{*}$. This is in fact the canonical Drinfeld-Sokolov construction. This foliation will be defined by an integrable subbundle of the tangent bundle of $\mathscr{M}$, and its corresponding space of leaves will be isomorphic to the dual of the Virasoro algebra. We will then check that the chosen subbundle satisfies enough conditions so as to guarantee the existence of a Poisson bracket on the space of leaves. This reduced bracket will be canonically induced by the Lie-Poisson bracket (1.4). The induced structure will also coincide with the Poisson structure on the dual of the Virasoro algebra.

Consider $\mathscr{M}$ to be the subset of $\hat{g}_{1}^{*}$ given by differential operators of the form

$$
P=-\frac{d}{d \theta}+\left(\begin{array}{cc}
\alpha & \beta \\
1 & -\alpha
\end{array}\right)
$$

In the general case of any semisimple Lie algebra $g$, one would consider a standard gradation for $g$ and define $\mathscr{M}$ as the set of differential operators of the form $-\frac{d}{d \theta}+\Gamma+L(\theta)$, where $\Gamma$ is a generator of the graded component of degree -1 and $L$ is a matrix function which takes values on the Borel subalgebra of $g$ corresponding to elements of nonnegative grading. In the $\operatorname{SL}(2, \mathbf{R})$ case the length of the grading is one, the negative graded part corresponds to strictly lower triangular matrices (with generator $\left(\begin{array}{ll}0 & 0 \\ 1 & 0\end{array}\right)$ ), and the Borel subalgebra is given by the upper triangular matrices, resulting in expression (2.1) above. 
The reason for restricting ourselves to the submanifold $\mathscr{M}$ is that an operator of the form (2.1) is equivalent to the second-order scalar differential operator

$$
L=\frac{d^{2}}{d \theta^{2}}+\left(\alpha^{\prime}-\alpha^{2}-\beta\right) \quad\left({ }^{\prime}=\frac{d}{d \theta}\right)
$$

in the sense that if $\xi$ is a solution of the equation $L \xi=0$, then the vector $v=\left(\xi^{\prime}+\alpha \xi, \xi\right)$ is a solution of the differential equation

$$
\frac{d v}{d \theta}=\left(\begin{array}{cc}
\alpha & \beta \\
1 & -\alpha
\end{array}\right) v .
$$

As it was shown in [4], the same assertion can be made for $\operatorname{SL}(n, \mathbf{R})$ and $\mathrm{GL}(n, \mathbf{R})$ where one finds an equivalence of elements of $\mathscr{M}$ with scalar differential operators of order $n$ of the form

$$
\frac{d^{n}}{d \theta^{n}}+u_{n-1} \frac{d^{n-1}}{d \theta^{n-1}}+\cdots+u_{1} \frac{d}{d \theta}+u_{0} .
$$

and where $u_{n-1}=0$ in the case of $\operatorname{SL}(n, \mathbf{R})$.

Therefore, if we wish to define an induced Poisson structure on the manifold of $n$ th-order scalar differential operators, we need to identify matrix differential operators of the form (2.1) which have the same scalar equivalent. Fortunately such an identification is easily achieved by factoring through the gauge action of certain subgroup of $G$, as next proposition shows.

Proposition 2.1 [4]. Let $P_{1}$ and $P_{2}$, respectively, be differential operators of the form

$$
P_{1}=-\frac{d}{d \theta}+\left(\begin{array}{cc}
\alpha_{1} & \beta_{1} \\
1 & -\alpha_{1}
\end{array}\right), \quad P_{2}=-\frac{d}{d \theta}+\left(\begin{array}{cc}
\alpha_{2} & \beta_{2} \\
1 & -\alpha_{2}
\end{array}\right) .
$$

Assume that they are equivalent to scalar differential operators $L_{1}, L_{2}$, respectively, in the sense mentioned above. Then $L_{1}=L_{2}$ if and only if there exists $g \in C^{\infty}\left(S^{1} ; \operatorname{SL}(2, \mathbf{R})\right)$ of the form

$$
g=\left(\begin{array}{cc}
1 & m \\
0 & 1
\end{array}\right)
$$

with $\operatorname{Ad}^{*}(g)\left(P_{2}\right)=P_{1}$.

That is, the identification we are looking for is equivalent to factoring through the gauge action of the (unipotent) subgroup of $\widetilde{G}$ consisting of elements of the form (2.2). We will call that subgroup $\tilde{N}$. In the general case one factors by the gauge action of the unipotent subgroup which corresponds to the Borel subalgebra that was initially chosen.

Proof. Assume $P_{1}$ and $P_{2}$ given as in the proposition and that $g$ is given as in (2.2), so that $\operatorname{Ad}^{*}(g)\left(P_{2}\right)=P_{1}$. From (1.2) we obtain that this condition is equivalent to

$$
m=\alpha_{1}-\alpha_{2}, \quad m^{\prime}=\beta_{1}-\beta_{2}+m\left(\alpha_{2}+\alpha_{1}\right) .
$$

And from this relation we can deduce that

$$
\alpha_{1}^{\prime}-\alpha_{1}^{2}-\beta_{1}=\alpha_{2}^{\prime}-\alpha_{2}^{2}-\beta_{2}
$$


so that $L_{1}=L_{2}$. And conversely, if $L_{1}=L_{2}$ then (2.3) holds and for

$$
g=\left(\begin{array}{cc}
1 & \alpha_{1}-\alpha_{2} \\
0 & 1
\end{array}\right)
$$

one gets $\operatorname{Ad}^{*}(g)\left(P_{2}\right)=P_{1}$.

Now that we know that the leaves of the foliation which we want to reduce coincide with the coadjoint orbits under the action of $\widetilde{N}$, we will give some conditions on the integrable subbundle of $T(\mathscr{M})$ that defines this foliation so that an induced Poisson structure can be canonically defined on the space of leaves. These conditions are given by (i)-(iii) below, and they are analogous to those stated in the theorem by Marsden and Ratiu in [10]. As we pointed out in the introduction, with a little extra work we could apply the theorem directly, but we prefer to repeat the argument to help the reader to understand better the geometry of the manifolds involved.

Let $\mathscr{M}$ be the subset of $\hat{g}_{1}^{*}$ given by elements of the form $(2.1)$. Let $E$ be the distribution generated by the Hamiltonian vector fields corresponding to the functionals $\left\{\varepsilon_{k}^{2}+\varepsilon_{k}^{3}\right.$, for all $\left.k\right\}$. Then conditions (i)-(iii) for $E$ are true.

(i) $E$ is an involutive distribution since $\left\{\varepsilon_{k}^{2}+\varepsilon_{k}^{3}, \varepsilon_{d}^{2}+\varepsilon_{d}^{3}\right\}(P)=0$.

Besides, if we denote that by $\delta \mathscr{H}$ the gradient of the operator $\mathscr{H}$ then, using the definition of the Lie-Poisson bracket

$$
\begin{aligned}
\left\{\varepsilon_{k}^{2}+\varepsilon_{k}^{3}, \mathscr{H}\right\}(P) & =\left(\delta \mathscr{H}, \operatorname{ad}^{*}\left(\delta\left(\varepsilon_{k}^{2}+\varepsilon_{k}^{3}\right)\right)(P)\right) \\
& =\left.\frac{d}{d t}\right|_{t=0}\left(\delta \mathscr{H}, \operatorname{Ad}^{*}\left(g_{t}\right)(P)\right)=\left.\frac{d}{d t}\right|_{t=0}\left(\mathscr{H} \circ \operatorname{Ad}^{*}\left(g_{t}\right)\right)(P),
\end{aligned}
$$

where $\mathscr{H} \in C^{\infty}\left(S^{1} ; \hat{g}_{1}^{*}\right), P \in \mathscr{M}$ and $g_{t}$ is a curve on $\tilde{N}$ such that $\left.\frac{d g_{t}}{d t}\right|_{t=0}=$ $\delta\left(\varepsilon_{k}^{2}+\varepsilon_{k}^{3}\right)$. The tangent space to the unipotent subgroup $\tilde{N}$ is contained in the subalgebra generated by $\delta\left(\varepsilon_{k}^{2}+\varepsilon_{k}^{3}\right)$, and, therefore the integral curves of $E$ generate the coadjoint orbits of the action of $\tilde{N}$ on $\mathscr{M}$.

Hence, the foliation defined by $E \cap T(\mathscr{M})$ is regular and $\mathscr{M} / \tilde{N}$ coincides with the space of leaves.

(ii) The second condition is that if $K$ and $L$ are smooth operators such that their differentials vanish on $E$, then the differential of the bracket $\{K, L\}$ should also vanish on $E$. That is, $E$ should leave $\{$,$\} invariant. This$ property trivially holds for $E$ since it is generated by Hamiltonian vector fields (if $\{f, h\}=\{f, g\}=0$, then $\{f,\{h, g\}\}=0$ by Jacobi's identity).

(iii) Finally, if we want the reduced bracket to be well defined we need the additional property

$$
\left\{E^{\perp}, \cdot\right\}(P) \subset T_{P}(\mathscr{M})+E_{P},
$$

for any $P \in \mathscr{M}$. A short inspection using the definition of the bracket shows that the vector space

$$
\left\{E^{\perp}, \cdot\right\}(P)=\left\{\{\mathscr{H}, \cdot\}(P):\left.\left\{\mathscr{H}, \varepsilon_{k}^{2}+\varepsilon_{k}^{3}\right\}\right|_{\mathscr{M}} \equiv 0, \text { for all } k\right\}
$$

is generated by the Hamiltonian vector fields corresponding to $\varepsilon_{d}^{2}+\varepsilon_{d}^{3}$ for all $d$, and, therefore, it coincides with $E_{P}$.

Now, for any smooth operators $f$ and $h$ on $\mathscr{M} / \tilde{N}$ define the bracket given by the formula

$$
\{F, H\} \circ i=\{f, h\}_{\mathscr{M} / \widetilde{N}} \circ \pi,
$$


where $i: \mathscr{M} \rightarrow \hat{g}_{1}^{*}$ is the inclusion, $\pi: \mathscr{M} \rightarrow \mathscr{M} / \tilde{N}$ is the natural projection, and $K$ and $H$ are any smooth extensions of $f \circ \pi$ and $h \circ \pi$, respectively, with differential vanishing on $E$.

Theorem 2.1. The bracket (2.4) is well defined and it induces a Poisson structure in the space of leaves $\mathscr{M} / \tilde{N}$. Furthermore, the space $\left(\mathscr{M} / \tilde{N},\{,\}_{\mathscr{M} / \widetilde{N}}\right)$ is Poisson isomorphic to a hyperplane of the dual of the Virasoro algebra with its canonical Lie-Poisson structure.

Before going into the proof of the theorem, we will recall the definition of the Virasoro algebra and the Lie-Poisson bracket on its dual and set some notation.

Let $\operatorname{vect}\left(S^{1}\right)$ be the algebra of vector fields on the unit circle, with the usual commutator of vector fields as Lie bracket. On vect $\left(S^{1}\right)$ define the so-called Gelfand-Fuks cocycle

$$
c\left(\xi \frac{\partial}{\partial \theta}, \mu \frac{\partial}{\partial \theta}\right)=\int_{0}^{2 \pi} \xi^{\prime} \mu^{\prime \prime} d \theta .
$$

The central extension obtained from $\operatorname{vect}\left(S^{1}\right)$ and $c$ is called the Virasoro algebra, and we will denote it by $g_{0}$. Its dual can be viewed as the set

$$
g_{0}^{*}=\left\{\left(p(\theta) d \theta^{2}, s\right), p(\theta) \text { a } 2 \pi \text {-periodic function, } s \in \mathbf{R}\right\}=\bigotimes^{2} S^{1} \oplus \mathbf{R},
$$

where $\otimes^{2} S^{1}$ represents the 2-tensor algebra on the circle. For more details see Witten [16] or Kirillov [8]. The Lie-Poisson structure on $g_{0}^{*}$ is, by definition,

$$
\{\mathscr{H}, \mathscr{L}\}_{0}(p, s)=\int_{0}^{2 \pi}\left(\delta \mathscr{H} \delta \mathscr{L}^{\prime}-\delta \mathscr{H}^{\prime} \delta \mathscr{L}\right) p(\theta) d \theta+s \int_{0}^{2 \pi} \delta \mathscr{H}^{\prime} \delta \mathscr{L}^{\prime \prime} d \theta,
$$

where here $\delta \mathscr{H}$ represents the gradient of $\mathscr{H}$ on the $p$-component. As it happened with the Kac-Moody algebra (and due to the central extension), $g_{0}^{*}$ stratifies into Poisson submanifolds corresponding to a fixed choice of the central parameter $s$; all of them are geometrically equivalent except for the case $s=0$. The hyperplane we are going to choose corresponds to the choice $s=-1$. Finally, if we denote by $\varepsilon_{k}(p)$ the $k$ th Fourier coefficient of $p$, the bracket (2.5) is written in $\varepsilon$-coordinates as

$$
\left\{\varepsilon_{k}, \varepsilon_{d}\right\}_{0}(p)=\frac{i}{2 \pi}(k-d) \varepsilon_{k+d}-\frac{i}{2 \pi} k^{3} \delta_{k+d}^{0} .
$$

Proof of Theorem 2.1. Conditions (i)-(iii) are going to guarantee that the bracket (2.4) is well defined. In fact, let $F$ be an extension of $f \circ \pi$, and let $H$ and $\widetilde{H}$ be two different extensions of $h \circ \pi$. Assume that the differentials of $F, H$, and $\widetilde{H}$ vanish on $E$. Then $d F \in E^{\perp}$, and it follows from $\{F, \cdot\} \in T(\mathscr{M})+E$ that $\{F, H\} \circ i$ is constant on the leaves of the distribution $E$. Moreover, $H-\widetilde{H}$ vanishes on $\mathscr{M}$ and the differential vanishes on $E ;\{F, H-\widetilde{H}\}_{P} \circ i=0$, and therefore the definition does not depend on the extension we choose.

Next, remember that each orbit can be identified with a periodic function $p(\theta)=\alpha^{\prime}-\beta-\alpha^{2}$ for some $\alpha$ and $\beta$ such that $P=-\frac{d}{d \theta}+\left(\begin{array}{ll}\alpha & \beta \\ 1 & -\alpha\end{array}\right)$ belongs to the orbit. This relation establishes an isomorphism between the space of leaves and $C^{\infty}$-functions on the circle. We will view the latter manifold as the underlying manifold for the hyperplane $s=-1$ in the dual of the Virasoro 
algebra, and we will show that their Poisson structures coincide. Fix $\left\{\varepsilon_{k}\right\}$, the $k$ th Fourier coefficients, as coordinates on $\mathscr{M} / \tilde{N}$.

Auxiliary Lemma 1. Let $P=-\frac{d}{d \theta}+\left(\begin{array}{cc}\alpha & \beta \\ 1 & -\alpha\end{array}\right)$, and let $\varepsilon_{k}$ be the kth Fourier coefficient of $\alpha^{\prime}-\beta-\alpha^{2}$. Then $\Upsilon_{k}$ given by

$$
\Upsilon_{k}(P)=i k \varepsilon_{k}^{1}(P)-\sum_{p} \varepsilon_{p}^{1}(P) \varepsilon_{k-p}^{1}(P)+\varepsilon_{k}^{2}(P)-\varepsilon_{k}^{3}(P)
$$

are extensions of $\varepsilon_{k} \circ \pi$ for all $k$. Furthermore, their differentials vanish on $E$ along $\mathscr{M}$.

Proof of Lemma 1. Let $X=\left(\begin{array}{cc}\alpha & \beta \\ 1 & -\alpha\end{array}\right)$. If we identify $\mathrm{sl}(2, \mathbf{R})$ with its dual through the Killing form on the algebra, the basis dual to $\left\{X_{1}, X_{2}, X_{3}\right\}$ is given by $X_{1}^{*}=2 X_{1}, X_{2}^{*}=-2 X_{2}$, and $X_{3}^{*}=2 X_{3}$. Therefore, $X$ can be written as

$$
X=2 \alpha X_{1}+\beta\left(X_{2}+X_{3}\right)+X_{3}-X_{2}=\alpha X_{1}^{*}+\frac{1}{2}(1-\beta) X_{2}^{*}+\frac{1}{2}(\beta+1) X_{3}^{*}
$$

so that we get the relations $\varepsilon_{k}(\alpha)=\varepsilon_{k}^{1}(X), \varepsilon_{k}(\beta)=\varepsilon_{k}^{3}(X)-\varepsilon_{k}^{2}(X)$, and $\varepsilon_{k}^{3}(X)+$ $\varepsilon_{k}^{2}(X)=\delta_{k}^{0}$, for any $X \in \mathscr{M}$.

It is now clear that

$$
\Upsilon_{k}(P)=i k \varepsilon_{k}^{1}(P)-\sum_{p} \varepsilon_{p}^{1}(P) \varepsilon_{k-p}^{1}(P)+\varepsilon_{k}^{2}(P)-\varepsilon_{k}^{3}(P)
$$

are extensions for $\varepsilon_{k} \circ \pi$ on $\hat{g}_{1}^{*}$. Besides, $\varepsilon_{k}^{2}+\varepsilon_{k}^{3}$ and $\Upsilon_{d}$ commute along $\mathscr{M}$ for all $k$ and $d$, and therefore the differentials of $\Upsilon_{k}$ all vanish on $E$ along

In order to finish with the proof of the theorem we need to show that the commutation relations for $\Upsilon_{k}$ are the same ones that we had above for the Virasoro algebra.

Following the definition (2.4), if $L \in \mathscr{M} / \tilde{N}$ and $\pi(P)=L$, we can show straightforward that $\{,\}_{\mathscr{M} / \tilde{N}}$ is given by

$$
\begin{aligned}
\left\{\varepsilon_{k}, \varepsilon_{d}\right\}_{\mathscr{R} / \widetilde{N}}(L)= & \left\{\Upsilon_{k}, \Upsilon_{d}\right\}(P) \\
= & \frac{i(d-k)}{2 \pi}\left[i(k+d) \varepsilon_{k+d}^{1}+\varepsilon_{k+d}^{2}-\varepsilon_{k+d}^{3}\right] \\
& +\frac{i k^{3}}{4 \pi} \delta_{k+d}^{0}+\frac{i}{\pi} \sum_{l}(k-l) \varepsilon_{k+d-l}^{1} \varepsilon_{l}^{1}
\end{aligned}
$$

Auxiliary Lemma 2.

$$
-\frac{i(d-k)}{2 \pi} \sum_{p} \varepsilon_{p}^{1}(P) \varepsilon_{k+d-p}^{1}(P)=\frac{i}{\pi} \sum_{p}(k-p) \varepsilon_{p}^{1}(P) \varepsilon_{k+d-p}^{1}(P),
$$

for all $P \in \mathscr{M}$.

Proof of Lemma 2. Assume $P=-\frac{d}{d \theta}+\left(\begin{array}{cc}\alpha & \beta \\ 1 & -\alpha\end{array}\right) \in \mathscr{M}$. Then $\varepsilon_{k}^{1}(P)$ is the $k$ th Fourier coefficient for the $2 \pi$-periodic function $\alpha$. It is easy to check that $(2.6)$ can be obtained from the fact that the $(k+d)$ th Fourier coefficients of $\frac{d \alpha^{2}}{d \theta}$ and $2 \alpha \frac{d \alpha}{d \theta}$ are equal. 
With the proof of this lemma we have obtained the following expression for the reduced bracket:

$$
\left\{\varepsilon_{k}, \varepsilon_{d}\right\}_{\mathscr{N} / \widetilde{N}}(L)=\frac{i(d-k)}{2 \pi} \varepsilon_{k+d}(L)+\frac{i k^{3}}{4 \pi} \delta_{k+d}^{0}
$$

which coincides with the Virasoro algebra structure. (Note: this structure is actually the negative of the Virasoro algebra structure. To have the exact coincidence we should have chosen $\varepsilon_{k}$ to be the $k$ th Fourier coefficient of $\beta+\alpha^{2}-\alpha^{\prime}$.)

Finally, some comments on transverse structures in infinite dimensions. The concept of transverse structure was initially introduced by Weinstein in [15] for finite-dimensional Poisson manifolds. The idea was to describe how the different symplectic structures induced on the coadjoint orbits are "put together" around a point which is singular for the Poisson tensor. One would choose an appropriate submanifold transverse to the symplectic leaves and would study (if possible) the Poisson structure induced canonically on it by the bracket of the manifold. It was proved in [15] that all transverse directions in a finitedimensional manifold are equivalent in the sense that one can find an isomorphism of the Poisson manifold taking the induced structure on one of the transverse submanifolds into the other one.

It is not hard to prove that, if we choose a linear section transverse to the orbit of maximal codimension, then a transverse structure for the bracket on $\hat{g}_{1}^{*}$ is given by the Lie-Poisson structure of $\mathrm{sl}(2, \mathbf{R})^{*}$. Besides, in [6] it was proved that the submanifold $\mathscr{M}$ is transverse to the coadjoint orbits, that is, symplectic leaves of both Poisson manifolds have the same codimension. In [11] we chose a linear section for the Virasoro algebra, transverse to the coadjoint orbit of the point $d^{2} / d \theta^{2}+n^{2} / 2$. The transverse structure found in [11] for the Virasoro algebra bracket was not linear, though linearizable and therefore equivalent to the Lie-Poisson bracket of $\operatorname{sl}(2, \mathbf{R})^{*}$. The reduction process is nonlinear in nature (due to the presence of $\alpha^{2}$ in the identification leaves $\leftrightarrow C^{\infty}$-functions on the circle). This implies that linear sections project onto nonlinear sections of the quotient manifold and vice versa. It is clear to us that this is a cause of the nonlinearity of the transverse structure in [11]. Notice also that, with the exception of $G=\operatorname{SL}(2, \mathbf{R})$ which corresponds to the Virasoro algebra, the second Gelfand-Dikii is always nonlinear. The fact that for the Virasoro algebra the final bracket is linear is certainly surprising.

Several interesting problems remain to be solved. It is reasonable to think that in the general case of the Second KdV Structure associated with a semisimple Lie group $G$, directions which are transversal to the leaves with $I$ and $-I$ monodromy can be found such that the transverse structure along them is equivalent to the Lie-Poisson structure on $g^{*}$. This can probably be solved using the definition (2.4) in the general case. It would also be very interesting to learn, using the geometry of these algebras, how the Poisson bracket behaves when we cross transversally coadjoint orbits which do not have maximal codimension.

\section{ACKNOWLEDGMENTS}

The author thanks Professor Jack F. Conn for introducing her to the subject of Poisson geometry and infinite-dimensional Lie algebras and offers special thanks to Professor Peter Olver for his support. 


\section{BIBLIOGRAPHY}

1. M. Adler, On a trace functional for formal pseudo-differential operators and the symplectic structure of the KdV, Invent. Math. 50 (1979), 219-248.

2. M. Antonowicz and A. P. Fordy, Hamiltonian structure of nonlinear evolution equations, Soliton theory: a survey of results, Manchester Univ. Press, New York, 1990.

3. P. Casati and M. Pedroni, Drinfeld-Sokolov reduction on a simple Lie algebra from a biHamiltonian point of view, Lett. Math. Phys. 25 (1992), 89-101.

4. V. G. Drinfel'd and V. V. Sokolov, Lie algebras and equations of KdV type, J. Soviet Math. 30 (1985), 1975-2036.

5. I. M. Gel' fand and L. A. Dikii, A family of Hamiltonian structures connected with integrable nonlinear differential equations, I. M. Gelfand, Collected Papers, vol. 1, Springer-Verlag, New York, 1987.

6. B. A. Khesin, Invariant of Hamiltonian KdV structures, Comm. Moscow Math. Soc. (1990).

7. T. G. Khovanova, The Gel'fand-Dikii bracket Lie algebras and the Virasoro algebra, Functional Anal. Appl. 20 (1986), 89-90.

8. A. A. Kirillov, Infinite dimensional groups: their representations, orbits, invariants, Proc. Internat. Congress of Math., Helsinki, 1978.

9. P. D. Lax, Integrals of nonlinear equations of evolution and solitary waves, Comm. Pure Appl. Math. 21 (1968), 467-490.

10. J. Marsden and T. Ratiu, Reduction of Poisson manifolds, Lett. Math. Phys. 11 (1986), 161-169.

11. G. Mari Beffa, A transverse structure for the Lie-Poisson bracket on the dual of the Virasoro algebra, preprint, 1991.

12. V. Yu. Ovsienko and B. A. Khesin, Symplectic leaves of the Gelfand-Dikii brackets and homotopy classes of nondegenerate curves, Funktsional Anal. i Prilozhen 24 (1990), 38-47; English transl. in Functional Anal. Appl. 24 (1990).

13. A. Pressley and G. Segal, Loop groups, Clarendon Press, Oxford, 1986.

14. A. G. Reiman and M. A. Semenov-Tjan-Sanskii, Current algebras and nonlinear partial differential equations, Soviet Math. Dokl. 21 (1985), 2.

15. A. Weinstein, The local structure of Poisson manifolds, J. Differential Geom. 18 (1983), 523-557.

16. E. Witten, Coadjoint orbits of the Virasoro group, Comm. Math. Phys. 114 (1988), 1-53.

Department of Mathematics, University of California at Davis, Davis, California 95616

Current address: Department of Mathematics, University of Wisconsin, Madison, Wisconsin 53706

E-mail address: maribeff@math.wisc.edu 